\title{
La Colección Hispánica (Siglo XVII) de Ediciones Colonienses en la Biblioteca de la Universidad de Illinois
}

\author{
JOSEPH L. LAURENTI \\ Illinois State University
}

Incide este trabajo de hoy en una serie de calas especializadas que venimos efectuando desde 1978 sobre colecciones de libros raros hispánicos impresos en diversas ciudades de Alemania durante la Edad Dorada que, actualmente, se albergan en la Biblioteca de la Universidad de Illinois, en Urbana, Illinois ${ }^{1}$.

Varios motivos nos llevan a recoger, y presentar aquí, estos fondos raros relativos a ciudades germánicas. La importancia histórica de estos fondos hispánicos del siglo XVII es especialmente significativa para el estudio de las relaciones culturales hispano-germanas del

1. Véanse, especialmente, las obras siguientes publicadas en colaboración con el colex:il .Alberto Porqueras Mayo: "Rarezas bibliográficas. Traducciones alemanas de libros de la Edad de Oro (siglos XVI y XVII) en la Universidad de Illinois", en Anmali dell' istituto Universitario Orientale - Sezione Romanza, vol. XX, n." 1 (1978), pp. 241-55; "Más sobre las traducciones alemanas de libros de la Edad de Oro (siglo XVII) ("Il li Biblioteca de la Universidad de Illinois", en Antonio Torres Alcalá, ed., Josep Maria Solà-Solé: Homage, homenaje, homenatge (Miscelánea de estudios de amigos y discípulos), Barcelona, Puvill, vol. II, 1984, pp. 255-63 y "La colección hispánica (siglo XVI) de ediciones colonienses de la Biblioteca de la Universidad de Illinois", en Varia Bibliographica, Homenaje a José Simón Díaz, Kassel Ed. Reichenberger, 1988. pp. 405-418. Véase, además, el artículo siguiente, por el autor de este trabajo de hoy, a saber: "La colección hispánica de impresos maguntinos (siglo XVI y XVII) en la Biblioteca de la Universidad de Illinois», en Boletín de la Biblioteca de Menéndez Pelayo, Año LXIII, (1987), pp. 333-351. 
Siglo de Oro. Los libros del Siglo de Oro se publican y traducen por todas partes de Alemania, aún durante la época de propaganda antiespañola en Alemania de 1580 a $1635^{2}$. El hecho de que muchos de estos libros se publicasen en latín aseguró su universalidad y el cruce de fronteras idiomáticas.

La colección aquí analizada representa nada más que un microcosmos de la colección hispánica de impresos alemanes de diversas ciudades germánicas. Se refiere, de momento, únicamente, por problema de espacio, a impresos colonienses, o sea a ejemplares raros impresos en la ciudad de Colonia entre 1602 y $1644^{3}$. Se trata, pues, de una colección que no sólo interesa al curioso lector por su importancia textual, por su valor crítico como pieza clave en la historia literaria, por sus ilustraciones, por su rareza, sino también por sus características tipográficas de impresos de una época en una ciudad determinada. Todo ello justifica, pues, la presentación de esta nueva colección de ejemplares de hoy.

Veamos, ahora, a guisa de presentación, algunas características que constituyen el perfil de esta colección hispánica de ediciones colonienses llegada a la biblioteca de la Universidad de Illinois.

Esta colección que describimos hoy se compone de nueve unidades bibliográficas. De éstas, hay que destacar la presencia de las inusitadas ediciones de la Institutionum dialecticarum libri octo y de la Isagoge philosophica (ficha n. ${ }^{\circ}$ 1) del jesuita Pedro de Fonseca, Colonia, Gosuinus Cholinus, 1605. Se trata de dos obras rarísimas de las que, de momento, además de los ejemplares de Urbana, sólo conocemos dos otros ejemplares en Inglaterra y Francia. El impresor Gosuinus Cholinus es hijo del impresor Maternus Cholinus, belga, que había impreso en Colonia desde 1577 a 1588 más de 350 obras. Maternus Cholinus mantuvo buenas relaciones con los jesuitas. De hecho, en 1586 imprime la primera edición de la Institutionum dialecticarum libri octo del padre Fonseca. La que presentamos en la ficha $n .^{\circ} 1$ es la segunda edición ${ }^{4}$.

2. Para más detalles sobre la época de propaganda antiespañola en Alemania véase Die trich Briesemeister, "Allerhand iniurien schmehkarten pasquill vnd andere schandlose ehrenrürige Schriften vnd Model. Die antispanischen Flugschriften in Deutschland 1580-1635", en Wolfenbütteler Beiträge, vol. 4 (1981), pp. 147-90.

3. Para los impresos colonienses anteriores al siglo XVII, véase «La colección hispánica (siglo XVI) de ediciones colonienses", citado en la nota anterior, n." 1 .

4. Para más detalles sobre los impresores Cholinus de Colonia es útil el capítulo corre's- 
Gran interés, por su extremada rareza, ofrece la versión latina de las Meditaciones de los misterios de nuestra Santa Fe (ficha n. ${ }^{\circ}$ 2), del P. Luis de la Puente (también jesuita), impresa en 1619 en el famoso taller de Juan Kinchius. Se trata de la tercera edición latina, única en Norteamérica y, al parecer, no presente en bibliotecas españolas.

El Padre Pedro de Rivadeneyra, S.J. publicó el Tratado de la tribulación... en castellano, en Madrid, en 1598. La obra contó pronto con traducciones a otras lenguas, especialmente al latín. En Urbana se encuentra la segunda edición alemana (la primera se publicó en Colonia, 1603) de Colonia, 1604 , (ficha $n .{ }^{\circ} 3$ ) de gran rareza, por cierto. La muestra urbanense es la única conocida en Norteamérica. Suponemos que sería fácil localizar algunos otros ejemplares más.

Capítulo aparte, por su extraordinaria importancia merecen las cuatro ediciones del jesuita español de Rueda, radicado en México, Antonio Rubio (fichas n. ${ }^{o s} 4,5,6$ y 7 ) $^{5}$. La ficha $n .^{\circ} 4$ representa la obra fundamental del P. Rubio, o sea la primera edición de su Comentariorum in universam Aristotelis logicam (Logica mexicana) impresa en 1605, por Arnold Birckmann, uno de los varios Birckmann, famosos impresores que existían en Colonia; incluso después de muertos, sus herederos seguían imprimiendo en sus prensas libros españoles usando el vago término de "Officina Birckmannica»". Esta edición del P. Rubio es de inusitada rareza en América y España. De momento, hemos localizado, además del ejemplar de Urbana, otros cinco ejemplares en diversas bibliotecas europeas.

Una de estas ediciones de singular rareza del P. Rubio es la tercera edición de De Anima (ficha n. ${ }^{\circ}$ ), impresa en 1613, por Johannes Crithius. El ejemplar de Illinois y los tres que se conservan respectivamente en el British Museum, en la Biblioteca Nacional de Ma-

pondiente a Colonia de Joseph Benzing, Die Buchdrucker des 16. und 17. Jahrhunderts im deutschen Sprachgebiet. Wiesbaden, Otto Harrassowitz, 1965.

5. Aunque breves avances de estas cuatro fichas fueron presentadas en mi articulo an. terior, a saber: "Antonio Rubio, S.J. (1548-1615) obras localizadas", Anuario de Letras (México), vol. XXIII (1985), pp. 299 310, estas entradas bibliográficas ahora corrigen y amplían considerablemente todos los datos presentados anteriormente sobre los impresos colonienses del P. Antonio Rubio. Por consiguiente en lo relativo a impre. sos colonienses del P. Rubio debe acudirse exclusivamente a la presente contribución.

6. Para otros libros españoles impresos por los diversos Birckmann, véase, en especial. el libro de Joseph Benzing, op. cit., pp. 224-226, 229, 233. 
drid y en la Biblioteca Bodleiana de Oxford, son, de momento, las únicas muestras conocidas en el mundo.

Del mismo impresor Johannes Crithius, la Universidad de Illinois posee una rarísima edición de los Comentarii vniversam Aristotelis dialecticam..., de 1621 (ficha n. ${ }^{\circ} 6$ ). De momento, sólo conocemos, aunque citado por Palau y Sommervogel, este ejemplar de la Biblioteca de la Universidad de Illinois. Es posible, pues, que este ejemplar sea uno de los muy pocos que se conserven en el mundo. Y, finalmente, destaca también por su rareza la octava edición del De Anima, de 1644, (ficha n. ${ }^{\circ}$ ), impresa también por Johannes Crithius. Se trata de una joya bibliográfica. Hemos localizado, además del ejemplar de Illinois, otro en la Biblioteca Carolina de Praga.

Mejor representada en Norteamérica y Europa es la Opera (ficha n. ${ }^{\circ}$ ) del prolífico humanista y antierasmista, de las cortes de Carlos V y Felipe II, Juan Ginés de Sepúlveda, impresa en 1602 en la Officina Birckmannica. De momento, conocemos, aunque citada solamente por Palau y Piacentini ${ }^{7}$, once ejemplares en ambos lados del Atlántico.

Por último la ficha $n .^{\circ} 9$ describe una rarísima edición, al parecer totalmente desconocida, y acaso el ejemplar de Illinois sea el único que se conserva en el mundo. Se trata de la versión latina de la $R e^{-}$ dención del tiempo cautivo del P. Franciscano Andrés de Soto, impresa en 1611 por Johannes Crithius. Las ediciones de esta obra, que tuvieron gran éxito, se repitieron profusamente, pero esta edición que presentamos hoy es la única que hemos localizado en Norteamérica y Europa.

Acabamos de presentar, pues, una colección de verdaderas joyas bibliográficas, de libros de autores españoles de la Edad de Oro en lengua latina e impresos en la ciudad alemana de Colonia. La mayoría de estos libros son de carácter religioso erudito, que solían imprimirse, precisamente, fuera de España, en busca de un mercado cosmopolita. Siete de estos libros son de jesuitas de América y España, cuya presencia en Illinois es abundantísima ${ }^{8}$.

7. Lástima que el denso y exhaustivo catálogo de Rafael Ramírez de Arellano, Ensayo de un catálogo biográfico de escritores de la provincia y diócesis de Córdoba (Madrid. 1925), págs. 611-25, no mencione esta obra de este autor cordobés.

8. Véanse, por ejemplo, las obras siguientes publicadas en colaboración cen $\Lambda$. Porcuri: ras Mayo: «La colección de Baltasar Gracián en la Biblioteca de la Universidad de Illi- 
Ojalá que con esta modesta aportación de hoy animemos a otros investigadores a efectuar calas como la presente para que, entre todos, lleguemos, algún día a recoger todas las ediciones alemanas de libros «sabios» hispánicos del Siglo de Oro y a localizar más ejemplares que se conservan en el mundo. Sólo así será posible estudiar a fondo el fascinante capítulo de la producción e influencia literaria de los autores del Siglo de Oro en Alemania y, al mismo tiempo, completar en lo posible los siempre clásicos y meritorios trabajos bibliográficos de Adam Schneider (op. cit.) y Werner Krauss (op. cit.).

\section{REPERTORIOS QUE SE CITAN ABREVIADAMENTE}

ANTONIO $=$ Nicolás Antonio, Bibliotheca hispana nova. 2. ${ }^{\mathrm{a}}$ edición, Madrid, Joaquín Ibarra, 1783-1788. 2 vols.

BERISTAIN = José Mariano Beristáin de Souza, Biblioteca hispanoamericana setentrional. 2. edición, publícala Fortino Hipolito Vera. Amecameca, Tip. del Colegio Católico, 1883-97. 4 vols.

CATALOGUE = Catalogue of the Printed Books in the Library of the University of Edinburgh: Volume III (P-Z). Printed by T. and Constable LTD. at the University Press, 1923.

CATALOGUS = Catalogus librorum impressorum bibliothecae bodleianae in academia oxoniensi: volumen tertium. Oxonii, E. Typographeo Academico, MDCCC.XLIII.

EGUIARA Y EGUREN = J.J. de Eguiara y Eguren, Bibliotheca mexicana sive eroditorum historia virorum... México, Ex Nova Typographia, 1755.

GANGA = Ginés Ganga, "Lista de obras de autores españoles de la Biblioteca de la Universidad Carolina de Praga», en Boletín de la Real Academia de la Historia, vol. 98 (1931), pp. 770-94.

nois: fondos raros (siglos XVII y XIX)», en Bulletin Hispanique, t. LXXIX, núms. 3-4 (1977), págs. 347-79; «La colección del P. Francisco Suárez, S.J. (1548-1617) (n la Bi. blioteca de la Universidad de Illinois", en Estudios Románicos dedicados al Prof. in. drés Soria Ortega, t. II Granada, Univ. de Granada, 1985, pp. 577-85; “la colección de. Juan Eusebio Nieremberg en la Universidad de Illinois (ediciones del siglo XVII)", (2II Philologica Hispaniensia in Honorem Manuel Alvar, t. III Literatura, Madrid, lidit. Gredos, 1986, págs. 355-68; y «La colección del P. Rivadeneyra, S.J. (1527-1611) en la Biblioteca de la Universidad de Illinois (siglos XVI y XVII)n, en Gutenberg Jahrbuch, 61 Jahrgang (1986), págs. 355-60. Véanse, además, las obras siguientes, del autor de este trabajo de hoy, a saber: "Antonio Rubio, S.J. (1548-1615): obras localizadas", (en Anuario de Letras, México, D.F., vol. XXIII (1985), págs. 299-310; "Presencia del P. Juan de Mariana, S.J. (1536-1624) en la Biblioteca de la Universidad de I!linois", (2II Renaissance and Golden Age Essays in Honor of D.W. McPheeters, Potomac, Maryland, Scripta Humanistica, 1986, págs. 148-66; "Jesuitas de los siglos XVI y XVII: impresos raros localizados", en Cuadernos bibliográficos, vol. 47 (1987), págs. 47-76 y «Martin del Río, S.J.: obras localizadas", en Anales de Literatura Española, n." 5 (1986)-1987), pp. 231-245. 
GRAESSE = Johann Georg Theodor Graesse, Trésor de livres rares et précieux. Dresde, 1859-69. 8 vols.

KRAUSS = Werner Krauss, Altspanische Drucke in Besitz der ausserspanischen Bibliotheken. Berlin, Akademie-Verlag, 1951. 1 vol.

LAURENTI-PORQUERAS = Joseph L. Laurenti y Alberto Porqueras Mayo, "Impresos raros de la Edad de Oro en la Biblioteca de la Universidad de Illincis, Letra F (Parte V)», en Anuario de Letras, vol. XIV (1976), pp. 179-216.

LAURENTI-PORQUERAS, GOLDEN AGE = Joseph L. Laurenti y Alberto Porqueras Mayo, The Spanish Golden Age (1472-1700). A Catalog of Rare Books Held in the Library of the University of Illinois and in Selected North American Libraries. Boston, G.K. Hall \& Co., 1979. 1 vol.

MEDINA = José Toribio Medina, Biblioteca hispano-americana (1493-1810). Santiago de Chile, En Casa del Autor, 1898-1907. 7 vol.

NUC $=$ The National Union Catalogue Pre-1956 Imprints, vols. 508 y 538. Mansell, 1977.

PALAU = Antonio Palau y Dulcet, Manual del librero hispanoamericano, tomos XVI, XVIII, XXI, XXII. Barcelona, Librería Palau, 1964, 1966, 1969-70.

PIACENTINI = Giuliana Piacentini, Repertorio del fondo antico spagnolo della biblioteca universitaria di Pisa. Pisa, Università, 1972. 1 vol.

SCHNEIDER = Adam, Spaniens Anteil an der deutschen Literatur des 16. und 17. Jahrhunderts von... Strassburg, I.G. Verlag von Schlefier \& Schweikhardts, 1898.1 vol.

SOMMERVOGEL = Auguste et Aloîs de Backer, S.J., Bibliothèque de la Compagnie de Jésus, lère partie: Bibliographie. 2me parte: Histoire, par le père Auguste Carayon. Nouvelle édition par Carlos Sommervogel. Bruxelles, O. Schepens \& Paris, A. Picard, 1890-1932. 11 vols.

\title{
SIGLAS CON QUE SE DESIGNAN \\ LAS BIBLIOTECAS EUROPEAS Y NORTEAMERICANAS
}

\section{A) Estados Unidos}

\author{
$\mathrm{CU}=$ University of California, Berkeley \\ $\mathrm{IU}=$ University of Illinois, Urbana, Illinois \\ MiU-C = University of Michigan - William L. Clements Library, Ann Arbor, \\ Michigan \\ $\mathrm{PU}=$ University of Pennsylvania, Philadelphia, Pennsylvania \\ $\mathrm{RPB}=$ Brown University, Providence, Rhode Island \\ RPJCB $=$ John Carter Brown Library, Providence, Rhode Island
}

\section{B) Europa}

$\begin{aligned} \text { EBU } & =\text { Edinburgh University Library, Edinburgh } \\ \text { GBM } & =\text { Bibliothèque Municipale, Grenoble } \\ \text { LBM } & =\text { British Museum, London } \\ \text { MBN } & =\text { Biblioteca Nacional, Madrid } \\ \text { OBB } & =\text { Bodleian Library, Oxford } \\ \text { PBN } & =\text { Bibliothèque Nationale, Paris } \\ \text { PBU } & =\text { Biblioteca Universitaria, Pisa }\end{aligned}$


[En este apartado de carácter presentativo y divulgativo, transcribimos, con exactitud, las portadas que aparecen en los originales. Cada ejemplar contiene una descripción minuciosa de la paginación o foliación del texto, del tamaño, de los grabados y de las signaturas internas de los pliegos. Al final de cada ficha, se consignan aquellos estudios críticos y noticias bibliográficas que versan sobre cada una de nuestras muestras o que apuntan sobre éstas noticias pertinentes.]

\section{FONSECA, Pedro de (1528-1599)}

1. PETRI FONSECAE / SOCIETATIS IESV. / INSTITVTIO - / NVM DIALECTICA-/ RVM LIBRI OCTO. / Emendatiùs quàm ante hac editi. / QVIBVS ACCESSIT / EIVSDEM AVTHORIS ISAGOGE / Philosophica, nunc demum in Germania / Cum librorum argumentis. Indice copio - / sissimimo \& capitum \& rerum. / 16 / [Escudo jesuitico, con el monograma IHS.] / 05. / COLONIAE, / Apud Gosuinum Cholinum, / [Filete] / Cum gratia \& priuilegio Caes. Maiest. /

$8 .^{0}-689$ p. nums. +18 hs. s. n. - Signs.: A- $\mathrm{Z}^{8}-\mathrm{Aa}-\mathrm{Zz}^{8}$.

Cits.: Laurenti-Porqueras, $n .^{\circ} 44$.

Ejemp.: IU, LBM, PBN.

La Isagoge Philosophica tiene portada propia.

Se trata de dos ediciones desconocidas por Sommervogel, tome II, cols. 838-40, que menciona únicamente la edición de 1611 (Colonia, Petrus Cholinus) y la de 1591 (Olysipone, Lisboa).

\section{PUENTE, Luis de la (1554-1624)}

2. MEDITATIONES / De /PRAECIPVIS FIDEI / NOSTRAE MYSTERIIS, VITAE AC / PASSIONIS D.N. IESV CHRISTI, ET. B. V. MARIAE, / Sanctorumq́; \& Euangeliorum toto anno occurrentium, / CVM ORATIONIS MENTALIS CIRCA E ADEM [sic] PRAXI. / AVTORE R. P. LVDOVICO DE PONTE VALLI-OLE - / tano Hispanicè editae, \& in duos Tomos diuisae. / INTERPRETE R. P. MELCHIORE TREVINNIO, VTROQVE / Societatis IESV Religioso. / HAC EDITIONE AVCTAE SVMMARIIS SEV SYNOPSI / Singularum Meditaionum. / [Grabado, con la leyenda: IHS DILECT.' ME.' QVEMADM. FIL' VNICORNIV. cant.] / COLONIAE AGRIPPINAE, / Apud IOANNEM KINCHIVM sub Monocerote, M.DC.XIX. / [Filete.] / Permissu superiorum, \& priuilegio. /

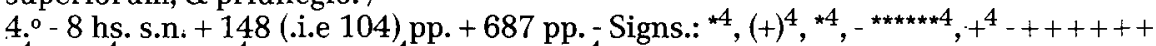
$+{ }^{4}, \mathrm{~A}-\mathrm{Z}^{4}-\mathrm{Aa}-\mathrm{Zz}^{4}-\mathrm{Aaa}-\mathrm{Zzz}^{4}-\mathrm{Aaaa}-\mathrm{Rrrr}{ }^{4}$.

Cits.: Schneider, p. 26; Sommervogel, tome VI, col. 1278.

Ejemp.: IU, LBM.

Tercera edición coloniense. Los ejemplares anteriores, impresos en Colonia (1611-12 y 1618), se albergan en la Nationalbibliothek de Viena y en la biblioteca Carolina, de Praga.

Muchos errores en la paginación del texto: pp. 66- 104 (primer grupo) están numeradas 510-516 y 117 -148; pp. 193-208 (segundo grupo) están numeradas 199 $-214$.

\section{RIVADENEYRA, Pedro de, S.J. (1527-1611).}

3. R.P. PETRI / RIBADENEIRAE, / SOCIETATIS IESV / PRESBYTERI, / DE TRIBVLATIONIBVS / huius seculi / Libri Duó. / IN QVIBVS DE OMNIBVS / humanae vitae miserijs, ac calamitatibus / agitur: veraque, ad salutares ex ijs, ani -/mae fructus colligendos, re - / media suggeruntur. / [Escudo jesuitico con el lema: LAVDABILE NOMEN DOMINI. IHS.] / [Filete] / COLONIAE, / Apud Coradum Butgenium. / M.DC. IIII. /

$12 .^{\circ}-6$ hs. s. n. +410 pp. - Signs.: ${ }^{* 6}, A-R^{12},-S^{2}\left(S_{2}\right.$ en blanco). 
Cits.: Catalogus, vol. III, p. 272; Ganga, p. 784; Graesse, vol. VI, p. 106; Palau, tomo XVI, n. ${ }^{\circ}$ 266303; Sommervogel, tome VI, p. 1733.

Ejemp.: IU, GBM, OBB, PBC.

Ejemplar adquirido a través del librero Mc Leish en 1951.

\section{RUBIO, Antonio, S.J. (1548-1615)}

4. LOGICA MEXICANA / siue / COMMENTARI / IN VNIVERSAM ARI - / STOTELIS LOGICAM. / Autore R. P. / Antonio Rvbio Rodensi, / Societatis Iesu Theologo, \& Professore in Regia Mexica - /norum Academia. / Cum duplici indice, quorum vnus quaestiones in parte discussas, al - / ter res consideratione dignas resert. / Pars Prior. / Quae quid contineat vide versa pagina. / Cum priuilegio S. Caes. Maiest. Superiorum permissu. / [Viñeta con un Cristo y el monograma IHS.]/Coloniae Agrippinae, / Sumptibus Arnoldi Mylii Birckmanni. / [Filete] / Anno M.DC.V./

$4 .^{\circ}-3$ hs. s. n. +1256 cols. +6 hs. s. n. - Signs.: ${ }^{*}, A-Z^{4}-\mathrm{Aa}-\mathrm{Zz}^{4}-\mathrm{Aaa}-\mathrm{Zzz}^{4}-\mathrm{Aaaa}$ - L $111^{4}$.

[A continuación, con paginación independiente y signaturas propias:]

LOGICAE MEXICANAE, siue / COMMENTARIORVM / IN VNIVERSAM ARISTO - / TELIS LOGICAM. / Autore R.P. / ANTONIO RVBIO RODENSI, / Societatis Iesu Theologo, \& Professore in Regia / Mexicanorum Academia. / PARS POSTERIOR. / Cum priuilegio S. Caes. Maiest. Superiorum Permissu. / [Viñeta con un Cristo y el monograma IHS.] / COLONIAE AGRIPPINAE, / Sumptibus Arnoldi Mylij Birckmanni / [Filete.] / Anno Domini M.DC.V. /

4. ${ }^{\circ}-792$ cols. - Signs.: A - $\mathrm{Z}^{4}-\mathrm{Aa}-\mathrm{Zz}^{4}-\mathrm{Aaa}-\mathrm{Ddd}^{4}$.

\section{[A continuación:]}

GENERALIS DISPV - / TATIO DE HABITIBVS, SEV / VERTVTIBVS INTELLECTVALI -/ bus ad partem Posterioristicam spectantibus. /

4. ${ }^{\circ} .210$ cols. +6 hs. de indices s. $n$. - Signs. $a-o^{4}-p^{2}$.

Cits.: Antonio, I, p. 159; Beristain, t. III, p. 71; Catalogue, III, p. 428; Catalogus, t. III, p. 326; Eguiara y Eguren, p. 269; Krauss, p. 85; Laurenti-Porqueras, Golden Age, p. 419; Medina, II, n. ${ }^{\circ}$ 505; NUC, vol. 508, p. 510; Palau, tomo XVIII, n. ${ }^{\circ}$ 280352; Sommervogel, tome VII, col. 280.

Ejemp.: IU, EBU, LBM, OBB, PBC, PBN.

La portada, que falta en el ejemplar ilinoyense, se ha suplido con la que describe Medina (opa . cit., pp. 57-58).

Se trata de la obra maestra del P. Rubio sobre el Organum de Aristóteles, que el autor intituló Logica mexicana, porque la escribió en México y en la Universidad de México la enseñó por varios años. La obra fue muy popular fuera de España. Esta edición es la primera publicada en Alemania.

5. R. P. / ANTONII / RVVIO RODENSIS, / DOCTORIS THEOLOGI SOCIETATIS / Iesu, S. Theologiae Professoris, / COMMENTARII / IN LIBROS ARISTOTELIS STAGIRITAE / Philosophorum Principis de Anima: vna cum dubijs \& quaestio - / nibus hac tempestate in scholis agitari soltis. / NVNC PRIMVN IN GERMANIA EDITI, CVM DV - / plici Indice, quorum vnus discussas omenes questiones alter res / omnes notables complectitur. / Ad. D.D. Ildephonsum De la Mota, Episcopum Tlaxcalensem, Re - / giumque Consiliarium. / PERMISSV SVPERIORVM / [Marca tipográfica, con el lema: RERVM VIGILANTIA CVSTOS.] / COLONIAE AGRIPPINAE. / Apud Ioannem Crithium sub signo Galli; ANNO M.DC.XIII./

$4 .^{\circ}-4$ hs. s. n. +542 pp. +11 hs. s. n.- Signs.: ${ }^{* 4}, \mathrm{~A}-\mathrm{Z}^{4}-\mathrm{Aa}-\mathrm{Zz}^{4}-\mathrm{Aaa}-\mathrm{Zzz}^{4}-\mathrm{Aaaa}^{4}$.

Colophon: COLONIAE AGRIPPINAE / Excudebat Petrus à Brachel./ Anno M.D.CXIII. / 
Cits.: Catalogus, vol. III, p. 326; Laurenti-Porqueras, Golden Age, p. 420; Palau, tomo XVIII, n. ${ }^{\circ}$ 280397; Sommervogel, tome VII, col. 283.

Ejemp.: IU, LBM, MBN, OBB.

El ejemplar de Illinois contiene una extensa dedicatoria del autor a D. Ildefonso de la Mota, obispo de Tlaxcala.

Nuestro ejemplar ingresó en julio de 1947, adquirido a través del librero Jeremy Norman de San Francisco.

6. R. P./ANTONII RVVIO / RODENSIS DOCTORIS / THEOLOGI, SOCIETATIS / IESV, / COMMENTARII IN VNIVERSAM / ARISTOTELIS DIALECTICAM: VNA CVM / dubiis \& quaestionibus hac tempestata agitari / solitis. / NVNC PRIMVM IN GERMANIA EDITI, CVM / Capitum, Dubiorum, Questionum, \& rerum notabilium, quae in / is continentur, INDICE. / PERMISSV SVPERIORVM / [Escudo con el monograma IHS.]/ COLONIAE, / Apud Ioannem Crithium, sub signo Galli./ [Filete.] / Anno Domini M DC. XXI /

4. ${ }^{\circ}-4$ hs. s. n. +530 pp. (i.e.539) +7 hs. s. n. - Signs.: $+^{4}, \mathrm{~A}-\mathrm{Z}^{4}-\mathrm{Aa}-\mathrm{Zz}^{4}-\mathrm{Aaa}-\mathrm{Zzz}^{4}$.

Cits.: Laurenti-Porqueras, Golden Age, p. 421; NUC, vol. 508, p. 510; Palau, tomo XVIII, n. ${ }^{\circ}$ 280348; Sommervogel, tome VII, col. 281.

Ejemp.: IU.

Edición rarísima. Acaso se trate del único ejemplar en el mundo. Es reproducción de la primera edición de Colonia, impresa en 1609 por Johannes Crithium. Nuestro ejemplar ilinoyense lleva el ex-libris de «W. H. Hadow. MCMH. Coll. Vicor. Oxon." en el verso de la portada que reza: «H.A.Pottinger, Worcester College, Oxford».

7. R. P. / ANTONII / RVVIO RODENSIS, / DOCTORIS THEOLOGI, SOCIETATIS / Iesu, S. Theologiae Professoris, / COMMENTARII IN LIBROS ARISTOTELIS / Stagiritae, Philosophorum Principis, / DE ANIMA / VNA CVM DVBIIS ET QVAESTIONIBVS IN / scholis hac tempestate agitari solitis, / CVM DVPLICI INDICE, VNO, CAPITVM, ALTERO / rerum notabilium. / PERMISSV SVPERIORVM./ [Marca tipográfica, con el lema: RERVM VIGILANTIA CVSTOS.]/ COLONIA AGRIPPINAE. / Apud Ioannem Crithium sub signo Galli; / ANNO 1644.

4. ${ }^{\circ}-1$ h. +512 pp. +11 hs. s. n. - Signs.: A - $Z^{4}-\mathrm{Aa}^{-} \mathrm{Zz}^{4}-\mathrm{Aaa}-\mathrm{Vvv}^{4}-\mathrm{Xxx}^{2}$.

Cits.: Ganga, p. 786; Krauss, p. 85; Laurenti- Porqueras, Golden Age, p. 420; NUC, vol. 508, p. 510.

Ejemp.: IU, PBC.

Edición rarísima, casi totalmente olvidada de todos los bibliógrafos y críticos de las obras del P. Rubio. La desconocen Palau, Medina, Sommervogel y Simón Díaz.

Se trata de una reproducción de la primera edición alemana del De Anima, impresa en Colonia en 1613 (véase ficha $n .^{\circ} 5$ ). Ambas se basan en la editio princeps, de 1611, de Alcalá de Henares, impresa por Andrés Sánchez Ezpeleta.

El ejemplar de Illinois lleva el ex-libris de la "Library of the society for promoting Christian knowledge».

\section{SEPULVEDA, Juan Ginés de (1490-1573)}

8. IOANNIS / GENESII / SEPVULVEDAE / CORDVBENSIS / Sacrosantae Theologiae Doctoris, Caroli V. / Imperatoris, Historici. / OPERA, / quae reperiri potuerunt/ OMNIA. / Quorum elenchum vide lector pagina quinta. / NVNC PRIMVM SINGVLARI STVDIO / in Hispania, Italia, \& Gallia ad publicam vtilitatem / conquisita, iam simul in lucem / edita. / Adiectus est copiosus rerum memorabilium index. / [Marca tipográfica, con la leyenda: VTILIA SEMPER NOVA SAEPIVS PROFERO.] / COLONIAE AGRIPPINAE, In Officina Birckmannica, sumptibus Arnoldi Mylij. / ANNO M DC II Cum gratia \& priuilegio Sac. Caes. Maiestatis. /

$4 .^{\circ}-6$ hs. s. $n .+634$ p. +8 hs. s. n. - Signs.: ${ }^{* 4}{ }^{* * 2}, \mathrm{~A}-\mathrm{Z}^{4} \mathrm{Aa}-\mathrm{Zz}^{4}-\mathrm{Aaa}-\mathrm{Zzz}{ }^{4}-\mathrm{Aaaa}$ $-\mathrm{Mmmm}^{4}-\mathrm{Nnnn}^{1}$. 
Cits.: Laurenti-Porqueras, Golden Age, p. 443; NUC, vol. 538, p. 527; Palau, tomo XXI, n. ${ }^{\circ}$ 309311; Piacentini, p. 159, n. ${ }^{\circ} 424$.

Ejemp.: IU, CU, LBM, MBN, MiU-C, PBN, PBU, PU, RPB, RPJCB.

Se trata de la primera edición completa de las trece obras del cordobés Juan Ginés de Sepúlveda. El ejemplar de Illinois contiene una biografía de Sepúlveda por Andrés Schott.

Nuestro ejemplar tiene el ex-libris de "Bibliotheca Acad. Ingolstadii».

\section{SOTO, Andrés de (¿1553? - 1625)}

9. CAPTIVI / TEMPORIS / REDEMPTIO. / LIBELLVS ORDINVM OM - / NIVM HOMINIBVS OMNI - / bus vtilissimus: in quo Temporis pre - / tium, vtque vinculis eripiendum / fit, declaratur: / PER REVERENDISS. P.F. / ANDRAEAM SOTO, ORDINIS / Francisci Reformatorum prouin - / ciae Conceptionis. / EPHES. V / Redimentes tempus quoniam dies ma - / li sunt. / [Escudo jesuitico con el monograma IHS y la leyenda: LAVDA BILE NOMEN DOMINI.] / COLONIAE,/ Apud Ioannem Crithium. / ANNO M.D.CXI. /

8. - 137 pp. +4 hs. s. n. - Signs.: A - $\mathrm{F}^{12} \cdot \mathrm{F}_{12}$ en blanco.

Cits.: Laurenti-Porqueras, Golden Age, p. 448; Palau, tomo XXII, p. 41.

Ejemp.: IU.

Versión latina de su Redención del tiempo cautivo. La primera versión en lengua española fue impresa en Amberes (Bellero) en 1606. La obra del P. Soto fue traducida al inglés por John Hawkins y publicada en Doway en 1634 por el impresor $G$. Pinsonl. 